\title{
Logic Verification of Incomplete Functions and Design Error Location
}

\author{
Qinhai ZHANG Charles TRULLEMANS \\ Laboratoire de Microélectronique, Université Catholique de Louvain \\ B-1348 Louvain-La-Neuve, Belgium
}

\begin{abstract}
At the stage of logic verification, it is necessary not only to detect but also to locate the sources of design errors that may exist in the gate-level circuit. For an incompletely specified function, a method to compute the corresponding 3-terminal BDD that represents the $\mathrm{ON}$-set, OFF-set and DC-set, is described. Two incomplete functions are equivalent if, and only if, their 3-terminal BDDs are isomorphic. If the gate-level circuit is verified to be incorrect, a conditional stuck-at fault model is proposed to represent the circuit with design errors. The incorrect logic values at the design error sites can be considered as conditional stuck-at faults. A design error locating method, based on fault simulation and released pattern generation, is described.
\end{abstract}

\section{Introduction}

Verification techniques[1-3] are often used to check the equivalence between the functional-level description and its gate-level implementation. Two complete functions are equivalent if, and only if, their 2-terminal BDDs are isomorphic[3]. For the verification of incomplete functions, the tautology checking[4], and the D-variable BDD[5] were proposed. In this paper, we describe a method to compute the 3-terminal BDDs from the given incomplete functions, and show that the 3-terminal BDDs maintain the canonical form even though complement edges are used. If the common don't care set is used, two incomplete functions are equivalent if, and only if, their 3terminal BDDs are isomorphic.

If a circuit is verified to be incorrect, the design errors that cause the misbehaviors of the circuit need to be found and corrected. A design error would occur, for example, by accidental substitution of one AND gate for an OR gate. For the location and correction of design errors, several works[6-10] have been reported. In this paper, a conditional stuck-at fault model is proposed. If a circuit is verified to be incorrect, a set of patterns that distinguish the circuit from its original description are generated. The design errors will cause some signal lines in the gate-level circuit to be faulty sources. When applying a distinguishing pattern to the circuit, some of these faulty sources can be considered as conditional stuck-at faults compared with the ideal circuit. If we only consider such design errors that cause single fault source, then the conditional stuck-at fault at the faulty source will be sensitized, and propagated to the primary outputs. A design error locating method, based on the fault simulation and released pattern generation, is described. The experimental results show that our method can locate the design error at one signal line in most cases.

In section 2, we first discuss the construction of a 3-terminal BDD from the 2terminal BDDs. We then show that the logic verification for two incompletely specified functions requires a simply comparison of their 3-terminal BDDs. In section 3 , we propose the conditional stuck-at fault model, then we describe the design error location method, and give some experimental results. 


\section{Verification of Incomplete Functions}

\subsection{A Review of BDDs}

Basic definitions of 2-terminal BDD and its implementation are given in $[3,11]$. With the fixed input variable ordering, a 2-terminal BDD is a canonical form for representation of a complete function. For any complete function, the corresponding 2-terminal BDD can be implemented by the recursive formulation of ITE(F, G, H) = $(F * G+\sim F * H)[11]$. For example, the function $F=a \oplus b$ can be represented as $F=$ $\operatorname{ITE}(a, \sim b, b)$, and its 2-terminal BDD is illustrated in Fig. 1a. If the complement edges are used, only the terminal 1 is necessary, and the canonical form can be maintained if all the "1" branches always keep the regular form[11]. In Fig. 1b, the compacted BDD of the function $F=a \oplus b$ is shown. A dot on an edge indicates a complement edge.

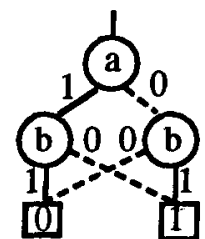

(a) Flat BDD

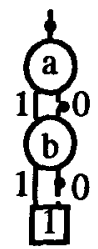

(b) Compacted BDD

Fig. 1. Flat and compacted BDDs of function $F=a \oplus b$

For representation of incomplete function with BDD, two methods are basically used. The first method uses the 3-terminal BDD, and expresses the don't cares explicitly, as shown in Fig. 2a. The second method encodes the incomplete function F into a pair of complete functions $\left[f_{0}, f_{1}\right]$. The $f_{0}$ and $f_{1}$ are the minimum and maximum function of $F$ when the don' $t$ care value ' $x$ ' is regarded as ' 0 ' and ' 1 ' respectively. This method combines the pair $\left[f_{0}, f_{1}\right]$ with an additional $D$-variable[5], and expresses the don't cares implicitly, as shown in Fig. 2b. We use the 3-terminal BDDs in this paper to represent incomplete functions.

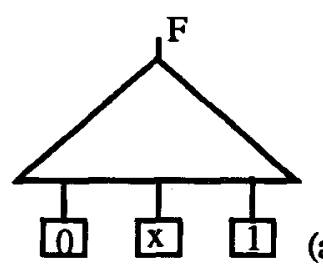

(a)

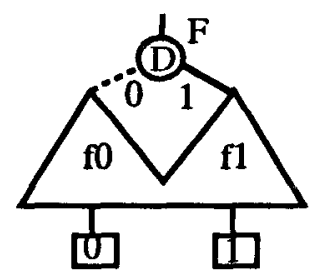

(b)

Fig. 2. (a) 3-terminal BDD (b) D-variable BDD

The sizes of BDDs for a given function are very sensitive to the input variable ordering[3]. An efficient method to compute the input variable ordering from a particular gate-level circuit, is described in [12]. The dynamic weight selection and intermediate node determination are used in that method to obtain the input variable ordering hierarchically. 


\subsection{Implementation of 3-Terminal BDDs}

By given the $O N$-set $F_{\text {on }}$ and the don't care set $F_{d c}$ of an incomplete function $F$, the 2-terminal BDDs of $F_{\text {on }}$ and $F_{d c}$ are implemented. Note that $F_{\text {on }}$ might be a gatelevel implementation of the incomplete function. Therefore, all the paths from the root of BDD Fon to the terminal " 1 " contain the complete ON-set and partial DCset( $\left(\mathrm{DC}_{\mathrm{On}}\right)$, and all the paths from the root of BDD Fon to the terminal " 0 " contain the complete OFF-set and partial DC-set(DC off $)$. Our aim is to extract the pure ON-set, OFF-set and DC-set and present them in one graph. For this purpose, an operator "\#" is introduced for the construction of the 3-terminal BDD F = Fon $\# F_{d c}$. This is illustrated in Fig. 3.
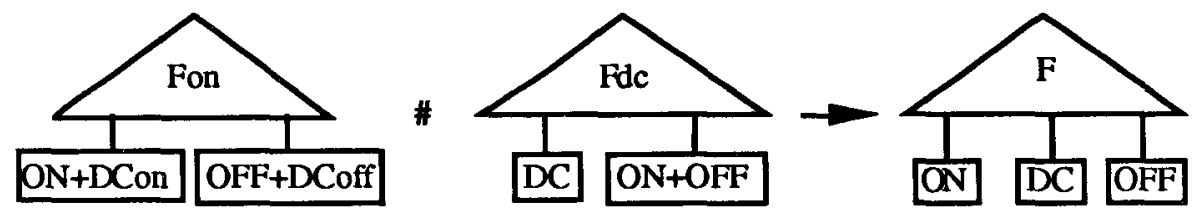

Fig. 3. Construction of the 3-terminal BDD $F$ from 2-terminal BDDs $F_{o n}$ and $F_{d c}$

The basic operation of "\#" is defined in Table 1 . If we use symbol " $x$ " for the terminal "DC", symbol " 1 " for the terminal "ON+DC 0 ", and symbol " 0 " for the

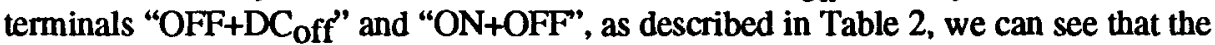
operator "\#" performs a partial function of the exclusive-OR operator " $\oplus$ ".

Table 1: Basic definition of the operator "\#"

\begin{tabular}{|c|c|c|}
\hline$F_{\text {on }} 1 F_{d c}$ & $D C$ & ON + OFF \\
\hline$O N+D C_{\text {on }}$ & $D C$ & ON \\
\hline OFF $+D_{\text {off }}$ & $D C$ & OFF \\
\hline
\end{tabular}

Table 2: the operator "\#" performs a partial function of the operator " $\oplus$ "

\begin{tabular}{|c|c|c|}
\hline $\mathrm{F}_{\mathrm{On}} 1 \mathrm{~F}_{\mathrm{dc}}$ & $\mathrm{x}$ & 0 \\
\hline 1 & $\mathrm{x}$ & 1 \\
\hline 0 & $\mathrm{x}$ & 0 \\
\hline
\end{tabular}

In our algorithm, the both 2-terminal BDDs of $F_{o n}$ and $F_{d c}$ are first implemented with the same terminal " 1 " in order to merge sub-functions. For representing an incomplete function, an additional terminal " $x$ " is needed to indicate the don't care cases. By using the $\operatorname{ITE}\left(\mathrm{F}_{\mathrm{dc}}, \sim \mathrm{F}_{\mathrm{on}}, \mathrm{F}_{\mathrm{on}}\right)$ for 3-terminal BDDs, two constraints are added. First, the node $v_{j}$ of the BDD Fdc is the first element of the triple. Second, a $\left[v_{j}, p_{j}\right]$ pair is used, where the $p_{j}$ is the path polarity from the root to the node $v_{j}$. The terminal cases are $\operatorname{ITE}\left([1,0], v_{i}, \sim v_{i}\right)=x$ and $\operatorname{ITE}\left([1,1], v_{i}, \sim v_{i}\right)=v_{i}$, where $v_{i}$ is a node of BDD Fon. Canonical representation form is a very important feature of BDD. To guarantee the canonical form of a 3-terminal BDD if complement edges are used, two additional standard triples must be added, in addition to the four standard triples described in [8]. These two additional triples are shown in Fig. 4. We always choose 
the right member of each equivalent pair. One example of constructing a 3-terminal $B D D F$ from the two 2-terminal BDDs $F_{\text {on }}$ and $F_{d c}$ is shown in Fig. 5.

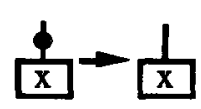

(a)

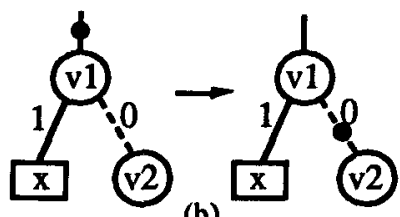

(b)

Fig. 4. Two additional standard triples
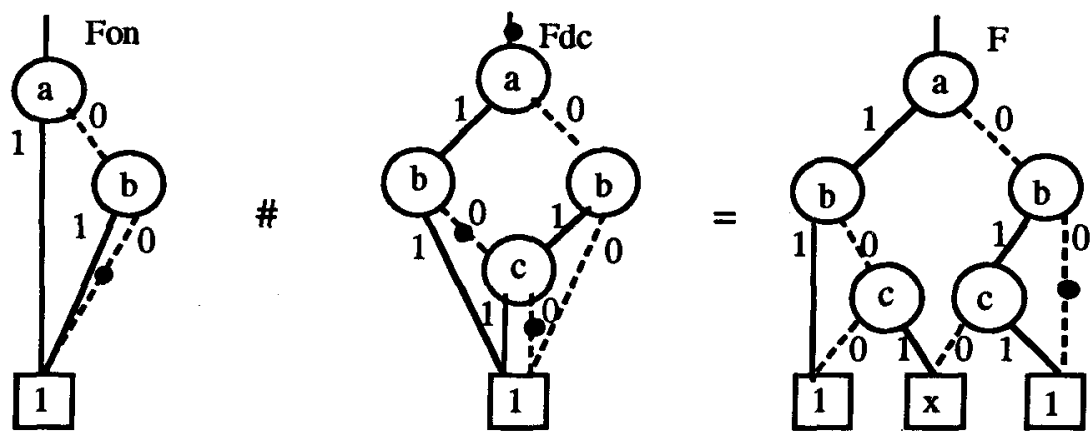

Fig. 5. An example of $F=F_{o n} \# F_{d c}$

\subsection{Experimental Results: Verification Based on 3-Terminal BDDs}

The verification of incomplete functions can be carried out by comparison the 3terminal BDDs. If the BDDs of function $F(G)$ is computed from the $F_{\text {on }}(G o n)$ and the common don't care set, i.e., $F=F_{\text {on }} \#\left(F_{d c}+G_{d c}\right)$, and $G=G_{o n} \#\left(F_{d c}+G_{d c}\right)$, the function $F$ is equivalent to $G$ if, and only if, their corresponding 3-terminal BDDs are isomorphic. Table 3 shows the experimental results on verification of incomplete functions for some circuits[16] compared with the tautology checking[4]. From lack of don't care sets of these circuits, two incomplete functions $F$ and $G$ are constructed for circuit $C$ as: $F_{o n}[i]=G_{d c}[i]=O_{c}[i]$, and $F_{d c}[i]=G_{o n}[i]=O_{c}[i-1]$. Since $F_{o n}[i] \epsilon$ $F_{d c}[i] \cup G_{d c}[i]$ and $G_{o n}[i] \in F_{d c}[i] \cup G_{d c}[i]$, the two incomplete functions $F$ and $G$ must be equivalent.

Table 3: Verification based on 3-terminal BDDs \& Tautology Checking

\begin{tabular}{|c|c|c|c|c|c|}
\hline \multirow{2}{*}{ Circuits } & BDDs Time & \multicolumn{2}{|c|}{ Verification Time } & \multicolumn{2}{c|}{ Maximum Memory } \\
\cline { 3 - 6 } & 3-T BDDs & Tautology & 3-T BDDs & Tautology \\
\hline C1355 & 1234.38 & 0.25 & 0.29 & 1453 & 1453 \\
\hline C1908 & 341.17 & 0.07 & 0.06 & 243 & 243 \\
\hline C432 & 185.78 & 7.08 & 8.27 & 162527 & 162527 \\
\hline C499 & 1197.75 & 37.25 & 30.23 & 22996 & 22995 \\
\hline C5313 & 46.54 & 67.32 & 22.75 & 9101 & 9824 \\
\hline C7552 & 52.56 & 94.27 & 197.80 & 19366 & 18848 \\
\hline C880 & 9.78 & 10.03 & 14.31 & 26722 & 31972 \\
\hline
\end{tabular}


The verification process is applied for each output separately. For a selected output, the 2-terminal BDDs $F_{o n}, F_{d c}, G_{o n}$, and $G_{d c}$ are first constructed. The CPU time is given in seconds on a DEC $5000 / 200$ workstation. The maximum memory is a measure of the maximum BDD node requirement, during the verification and BDDs implementation. The results show that our algorithm, for the verification of two incompletely specified functions, is comparable with the tautology checking with respect to the CPU time and memory requirement.

\section{Design Error Location}

\subsection{Distinguishing Pattern Generation}

For the purpose of locating design errors, a pre-simulation process is used. Both the functional-level description $F$ and gate-level circuit $G$ are first simulated with 64 random patterns. Once an erroneous output has been found, the pre-simulation process is terminated, and the formal verification for that output begins in order to generate the complete distinguishing pattern set. If no erroneous output has been found, the BDD verification then performs on each output separately.

For a selected erroneous output $O_{j}$, the function $D i f=F_{i} \oplus G_{i}$ is defined as a difference function. All the paths from the root of Dif to the terminal 1 contribute to the set of distinguishing patterns. If the functional-level description $\mathrm{F}$ is not available, the set of simulation patterns given by the designer can be used as a partial functional description, and all the unspecified patterns are treated as don't cares.

\subsection{Conditional Stuck-at Fault Model}

Design errors may exist in the gate-level design due to the accidental introduction by the designer, or to the undiscovered bugs in the logic synthesis software. In practice, the most common design errors are simple errors, namely, substitution of one simple gate for another, simple extra or missing gate errors, extra or missing wire errors, exchange wire errors, etc.[1]. Design errors can be classified into two classes: (I) the design errors only cause a signal line to be faulty source; (II) the design errors make two or more signal lines to be faulty sources. Most of the simple design errors are class-I design errors. Fig. 6 shows some simple design errors.

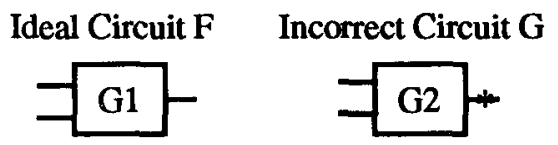

(a) Gate substitution

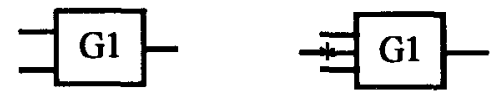

(b) Extra wire

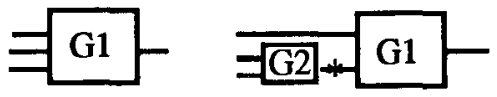

(c) Extra gate

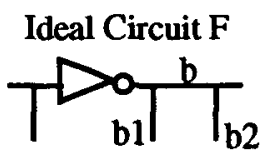

(d) A missing inverter
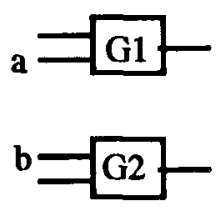

Incorrect Circuit $\mathbf{G}$
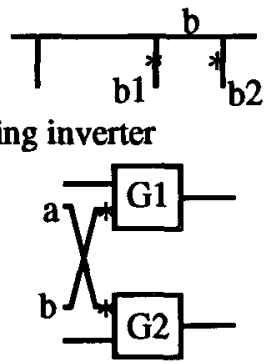

(c) Wire exchange

Fig. 6. Some simple design errors 
The '*' symbols marked at the incorrect circuits indicate the positions of faulty sources. The complex design errors and multiple design errors usually create multiple faulty sources, and are taken into account in class-II design errors. The location of the sources of class-II design errors is much more difficult than class-I design errors. We assume that only single class-I design error in the circuit is considered.

For analyzing the function of the circuit and locating the sources of design errors, a conditional stuck-at fault model is proposed. Assume that the functions of signal line $h$ in the ideal circuit $F$ and the incorrect circuit $G$ are $h_{f}(x)$ and $h_{g}(x)$ respectively. For a given input pattern $v$, a conditional stuck-at- $h_{g}(v)$ fault is said to exist at the signal line $h$ if $h_{f}(v)=-h_{g}(v)$. Notes that the widely used stuck-at fault model assumes that a signal line $h$ is stuck-at " 0 " or " 1 " permanently. In contrast, the design errors within a circuit make some signal lines to be temporarily, or conditionally stuck at " 0 " or " 1 " under certain patterns. For example, in Fig. 7, a design error exists by substituting the OR gate in the ideal circuit(a) for a NAND gate in the incorrect circuit(b). The two circuits are not equivalent under the patterns $[a / 1, b / 1, c / 1]$ and $[a / 1, b / 0, c / 0]$. For the first pattern, the signal lines $h$ and $o$ in the incorrect circuit(b) seem to be both stuckat -0 , and for the second pattern, the same signal lines $h$ and 0 seem to be stuck-at- 1 .

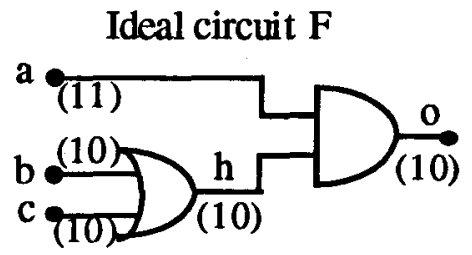

(a)
Incorrect circuit $\mathbf{G}$

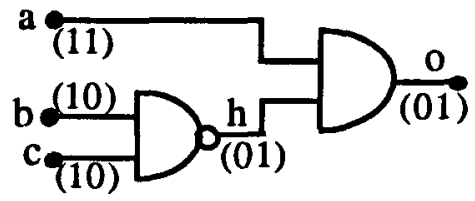

(b)

Fig. 7. One example of conditional stuck-at fault

\subsection{Design Error Location}

Assume that the signal line $h$ is the faulty source, and its associated functions in the circuits $F$ and $G$ are $h_{f}(x)$ and $h_{g}(x)$ respectively. The Shannon expansions of functions $F$ and $G$ with respect to the internal variable $h$ are defined as:

and

$$
\mathrm{F}(\mathrm{x})=\mathrm{h}_{\mathrm{f}}(\mathrm{x}) * \mathrm{~F}_{\mathrm{hf}=1}(\mathrm{x})+\sim_{h_{\mathrm{f}}(\mathrm{x})} * \mathrm{~F}_{\mathrm{hf}=0}(\mathrm{x}) \quad \ldots(3-1)
$$

$$
\mathrm{G}(\mathrm{x})=\mathrm{h}_{\mathrm{g}}(\mathrm{x}) * \mathrm{G}_{\mathrm{hg}=1}(\mathrm{x})+\sim_{\mathrm{h}}(\mathrm{x}) * \mathrm{G}_{\mathrm{hg}=0}(\mathrm{x}) \ldots(3-2)
$$

If considering the internal variable $h$ as an independent variable, the function $F^{*}(x, h)$ must be equivalent to the function $G^{*}(x, h)$, because the difference between functions $F(x)$ and $G(x)$ only results from the difference of $h_{f}(x)$ and $h_{g}(x)$. That is, $F_{h f=1}(x)=$ $G_{h g=1}(x)$, and $F_{h f}=0(x)=G_{h g=0}(x)$. Thus, from (3-1) and (3-2), we have

$$
\text { Dif }=\left[h_{f}(x) \oplus h_{g}(x)\right] * d G^{*}(x, h) / d h \quad \ldots(3-3)
$$

Where $\mathrm{dG}^{*}(\mathrm{x}, \mathrm{h}) / \mathrm{dh}=\left[\mathrm{G}_{\mathrm{hg}=1}(\mathrm{x}) \oplus \mathrm{G}_{\mathrm{hg}=0}(\mathrm{x})\right]$ represents the Boolean difference of circuit $G$ with respect to the internal variable $h$.

Theorem 3.1: A signal line $h$ is said to be the faulty source if, and only if, the following two conditions are satisfied for each distinguishing pattern v: (a) the 
conditional stuck-at- $h_{g}(v)$ fault is detected, i.e., $\mathrm{dG}^{*}(\mathrm{v}, \mathrm{h}) / \mathrm{dh}=1$; (b) the conditional stuck-at- $h_{\mathrm{g}}(\mathrm{v})$ fault is excited, i.e., $\mathrm{h}_{\mathbf{f}}(\mathrm{v}) \oplus \mathbf{h}_{\mathrm{g}}(\mathrm{v})=\mathbf{1}$.

Proof. The theorem holds directly from the equation (3-3).

By the condition (a) of theorem 3.1, any signal line $h$ that satisfies $\mathrm{dG}^{*}(\mathrm{x}, \mathrm{h}) / \mathrm{dh} \supseteq$ Dif, is a candidate of the faulty source. Given a distinguishing pattern $v$, the detectability of the conditional stuck-at-h $\mathrm{h}_{\mathrm{g}}(\mathrm{v})$ fault at the signal line $\mathrm{h}$ can be easily determined by fault simulation. However, the signal lines that are on the sensitive paths and propagate the fault effect to the outputs, also satisfy the condition. In order to determine such signal lines, we give the following theorem.

Theorem 3.2: Assume that there are $k(k \geq 1)$ fan-ins of signal line $b$ that have the controlling values for a distinguishing pattern $v$. If there is a pattern $u, u \in$ $d G^{*}(x, h) / d h$, but $u \notin D i f+D C_{i}\left(\right.$ the don't care set of output $\left.O_{i}\right)$, and if there are also $k$ fan-ins that have the controlling values with the pattern $u$, the signal line $h$ is not the faulty source. Such a pattern $u$ is called a released pattern to the signal line $h$.

Proof. Assume that the function $h_{f}$ of signal line $h$ is a symmetric function of its fan-ins, $h_{\mathbf{f}}\left(x_{1}, x_{2}, \ldots, x_{i}, x_{j}, \ldots, x_{m}\right)=h_{f}\left(x_{1}, x_{2}, \ldots, x_{j}, x_{i}, \ldots, x_{m}\right)$. This assumption is suitable for all the basic gates. Let the signal line $h$ be the faulty source. Since there are the same number of fan-ins that have the controlling values for both patterns $v$ and $u$, we have $h_{f}(v)=h_{f}(u)$, and $h_{g}(v)=h_{g}(u)$. Therefore, the pattern $u$ also excites the stuck-at-h $h_{g}(v)$ fault at the signal line $h$, i.e., $h_{g}(u) \oplus h_{f}(u)=1$. Since $u \in$ $\mathrm{dG}^{*}(\mathrm{x}, \mathrm{h}) / \mathrm{dh}$, the pattern $\mathrm{u}$ must be a distinguishing pattern according to the theorem 3.1. This is contradictory to $u \notin$ Dif.

For locating the faulty source that lies somewhere within the circuit, two steps are used. First, the set of signal lines that satisfy $\mathrm{dG}^{*}(x, h) / d h ~ \supseteq$ Dif is computed by fault simulation. The initial set of candidates includes all the signal lines that are the transitive fan-ins of current erroneous output. For each distinguishing pattern $v$, the circuit is first simulated. Then for each signal line $h$ in the candidate set, a stuck-at$\sim h_{g}(v)$ fault is injected, and fault simulation is performed. If the fault is detected, the signal line $h$ remains a candidate, otherwise the signal line $h$ is dropped. After the fault simulation, the candidate set usually contains more than one signal lines. By the theorem 3.2, we can further reduce the candidate set by released pattern generation. If there is a released pattern for the signal line $\mathrm{h}$, it can be dropped from the candidate set. This technique is very efficient for eliminating the candidates that are only on the sensitive paths.

As an explanation of our method, one simple circuit TR is used. The functionallevel description $F$ written in Boolean equations is given in Fig. 8a, the net-list $G$ is shown in Fig. 8b, and no don't care exists. The variable ordering is computed as \{ 13 , I4, 17, I1, I2, I8, I5, 16\}, and the BDD of Dif is shown in Fig. 8c. 11 distinguishing patterns were generated.

By performing the fault simulation with these distinguishing patterns, only the fanout stems that are in the current candidate set, are needed to be explicitly simulated. For example, at the pattern labeled at Fig. 8b, a conditional stuck-at-0 fault at the fanout stem $M 2$ can be detected, $M 2$ remains a candidate, the signal lines $\{I 1, I 2, M 1, I 7$, I3, I4, I8\}, and the fan-out branch \{M2-M5\} are dropped from the set of candidates. After the fault simulation, the candidate set contains the signal lines \{O0, M4, M2'\} 
and the fan-out branch (M2-M2'\}. By released pattern generation, we can find a released pattern [I1/x, I2/x, I3/1, I4/x, I5/1, I6/x, I7/0, 18/1] for both signal lines $\mathrm{O} 0$ and M4. Then, the signal lines $\mathrm{O} 0$ and M4 are dropped. The final candidate set contains the signal line \{M2'\} and the fan-out branch (M2-M2'\}. Notes that the signal line M2' and the fan-out branch M2-M2' are error equivalent sites.

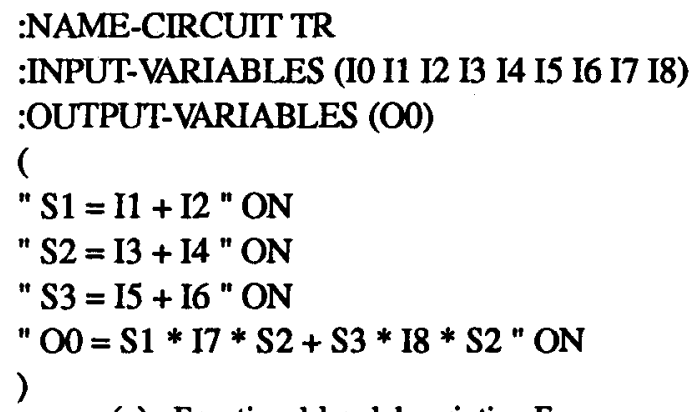

(a). Functional-level description $\mathrm{F}$
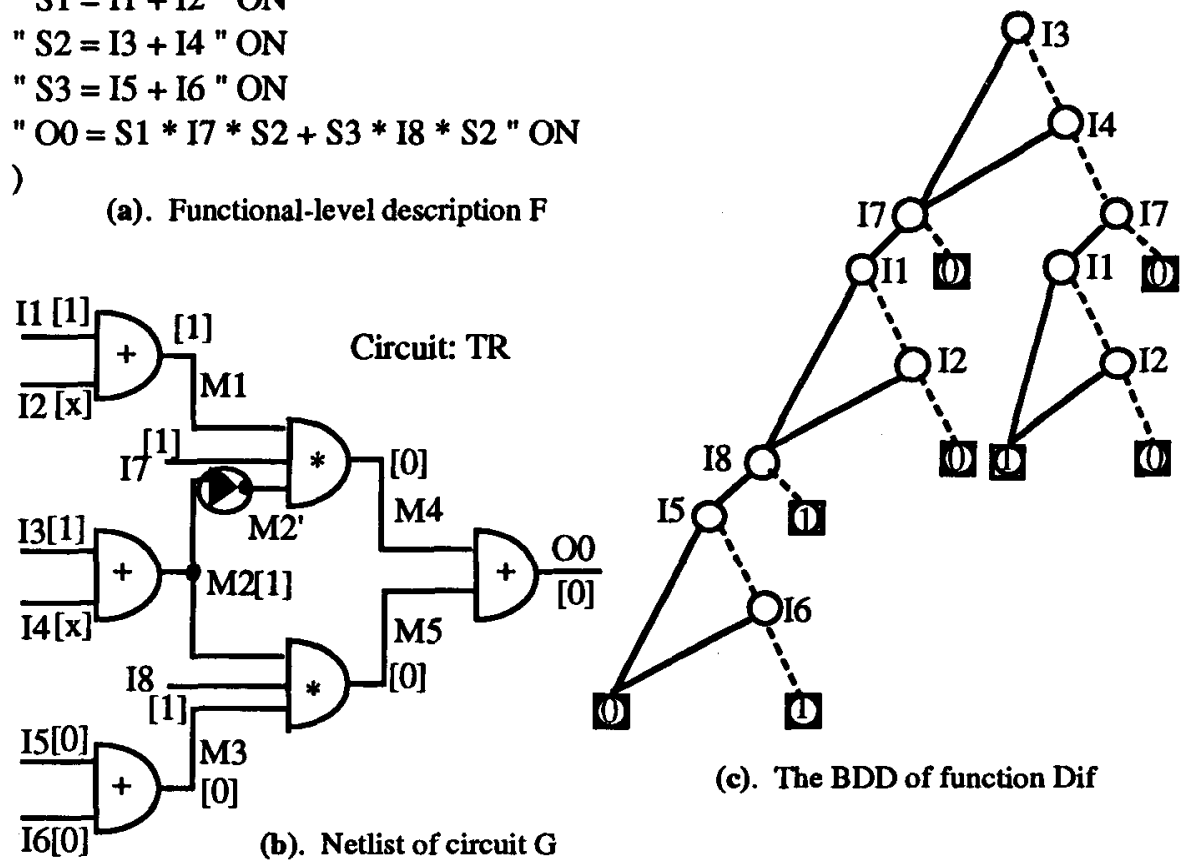

(c). The BDD of function Dif

(b). Netlist of circuit G

Fig. 8. An example of design error location

\subsection{Hybrid Fault Simulation and Released Pattern Generation}

Any combinational circuit can be partitioned into fan-out free regions(FFR)[13]. With FFRs, the expensive fault simulation of combinational circuits can be restricted to the explicit simulation of fan-out stems, and the detectability of other faults are determined locally inside the FFRs[13]. Critical path tracing[14] deals with the faults only implicitly, no fault dropping is necessary. However, the narrowing of faulty candidates is required for the design error location. In our method, the explicit fault simulation is only performed on the fan-out stems that are in the current candidate set.

Since some primary inputs may be unspecified in a distinguishing pattern, the BDDs are used as symbolic representations of signal lines in logic simulation. With BDD technique, a fault injection can be introduced to the objective signal line by 
complementing the related BDD. The fault is said to be detected if, and only if, the output BDD of the circuit is also complemented.

After the fault simulation, the final candidate set usually contains several candidates. In fact, most of them are only on the sensitive paths, and propagate the fault effect to the output. For eliminating such candidates, released pattern generation, which is analogous to test pattern generation[15], is introduced. However, there are two main differences. First, the released pattern does not fall into the don't care set and distinguishing pattern set. For this limitation, the BDD of Dif acts as a guidance for the input variable decision making, during the released pattern generation. Second, the released pattern not only detects a conditional stuck-at fault at the signal line $h$, but also set the same number of fan-ins of signal line $h$ with the controlling values as previously simulated distinguishing patterns.

\subsection{Speed up Techniques}

For a complex circuit, the complete set extracted from the BDD of Dif is very large, and then the fault simulation with these distinguishing patterns either takes a large fraction of computing time, or becomes impossible. In order to speed up the design error locating process, two heuristics are described in this sub-section.

Static Intermediate Variables: If there is a static intermediate variable(SIV)[12] in a circuit, the circuit can be divided by this SIV into two parts as shown in Fig. 9a. Since all the combinations of $X 1$ will set the logic value of the SIV M to 0 or 1 , the SIV $M$ can be used as a pseudo primary input to stand for all the inputs X1.

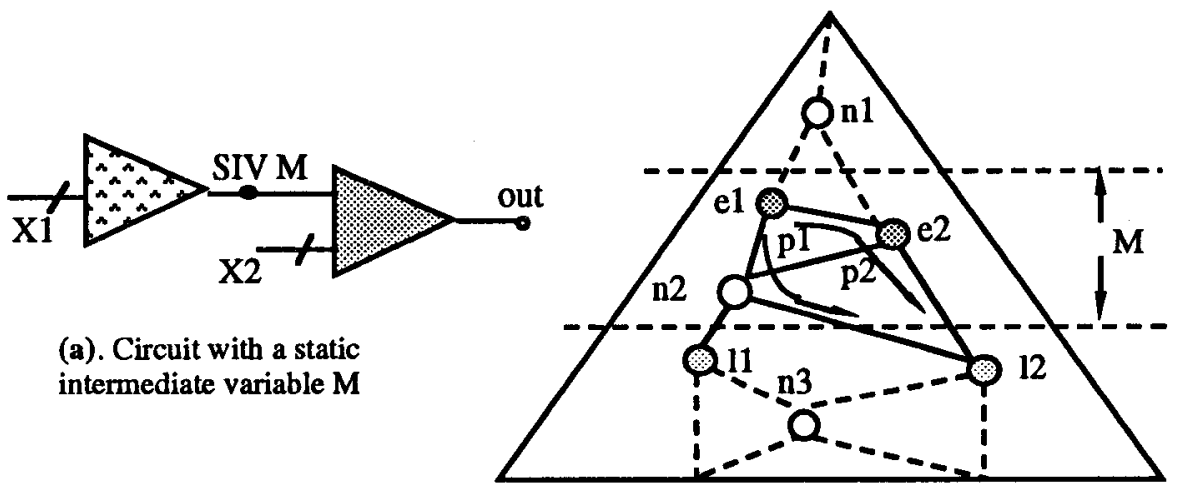

(b). Entering nodes and leaving nodes

Fig. 9. SIV nodes and the corresponding sections in the BDD

According to the strategy of input variable ordering[12], a subset of input variables covered by a SIV $M$ are grouped and corresponding to a section of Dif. For a path from the root of Dif to the terminal 1 , an entering node is defined as the first node located in the section, and a leaving node is defined as the first node out of the section. As shown in Fig. $9 \mathrm{~b}$, the nodes $\mathrm{e} 1$ and $\mathrm{e} 2$ are entering nodes of section $\mathrm{M}$, the nodes 11 and 12 are the leaving nodes, and $n 1, n 2$ and $n 3$ are neither entering nodes, nor leaving nodes. A triplet $\left[e_{i}, l_{j}, v_{i j}\right]$ is used to indicates the logic value $v_{i j}$ of a SIV for 
all the paths between an entering node $e_{i}$ and a leaving node $l_{j}$. If there are Pa paths from the root of the $\mathrm{BDD}$ Dif to the entering node $\mathrm{e}_{\mathrm{i}}, \mathrm{Pb}$ paths from $\mathrm{e}_{\mathrm{i}}$ to the leaving node $\mathrm{l}_{j}$, and $\mathrm{Pc}$ paths from $\mathrm{l}_{\mathrm{j}}$ to the terminal 1 , then a total of $\mathrm{Pa} *(\mathrm{~Pb}-1) * \mathrm{Pc}$ distinguishing patterns can be reduced by using the triplet $\left[e_{i}, l_{j}, v_{i j}\right]$. The SIVs and their covered primary inputs can be found in the ordering tree[12]. Fig. 10a shows the ordering tree for the circuit shown in Fig. 8. The nodes M2, M1 and M3 are SIVs. We first select the highest level SIVs $\{M 2, M 1, M 3\}$ to replace the covered input variables in the corresponding sections. The modified Dif is shown in Fig. 10b. By using the SIVs, seven distinguishing patterns can be saved. However, if a SIV node remains in the final candidate set, the design error location process for the corresponding sub-circuit is then continued.

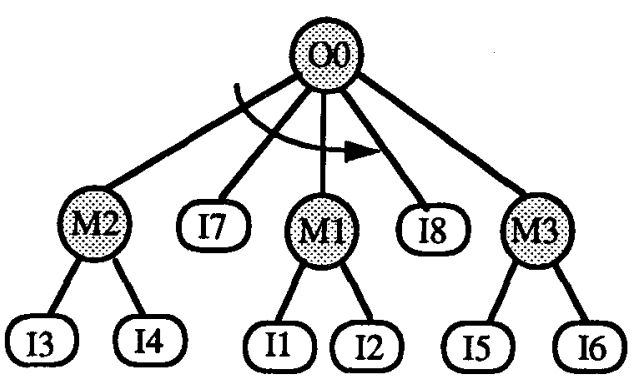

(a). Variable ordering tree

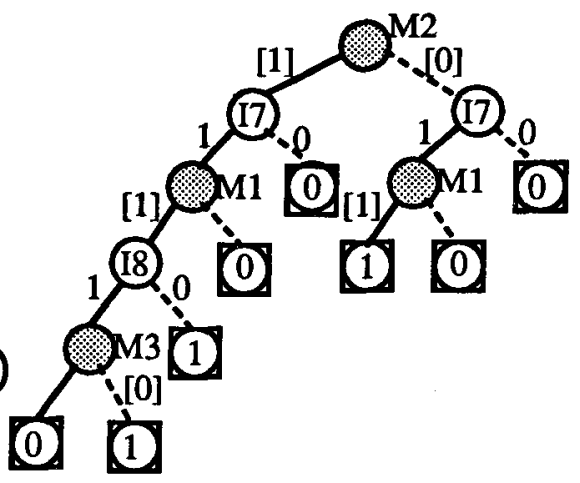

(b). The modifed Dif with [ei, lj, vij] triplets

Fig. 10. Variable ordering tree and modified BDD Dif

Fault Simulation with Partial Distinguishing Patterns: It has been observed that the size of candidate set rapidly decreases in the case of applying a small number of distinguishing patterns, then, it slowly decreases as shown in Fig. 11. Therefore, to instead of simulating all the distinguishing patterns, we choose a number of distinguishing patterns for fault simulation. When the size of candidate set reaches an appropriate value, the fault simulation is terminated, and the released pattern generation is then used for further reducing the candidate set.

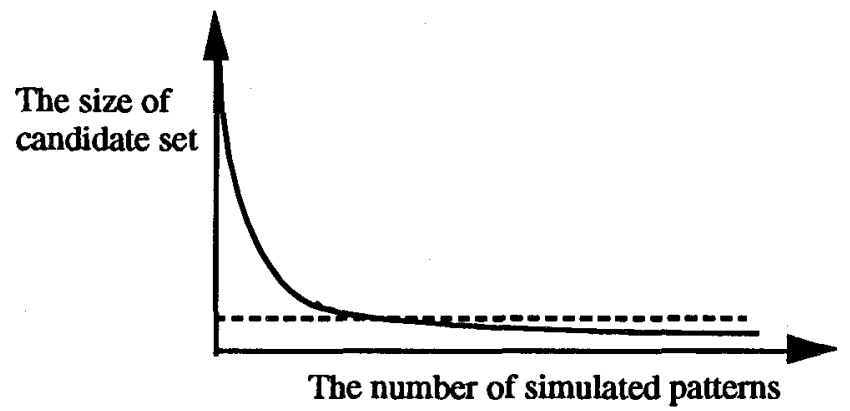

Fig. 11. The size of candidate set vs. the simulated patterns 


\subsection{Experimental Results: Design Error Location}

Dedia, a design error locating tool, based upon the method described above was implemented with $C$ language on a DEC $5000 / 200$ workstation. The inputs of Dedia are the functional-level description(Boolean equations), the don't care set and the netlist representation of the gate-level implementation.

A number of different class-I design errors are artificially introduced into the net-list representations. These different design errors inserted to the circuits are described in Table 4. The first two circuits are real designs in our laboratory, and the others come from the ICCAS85 benchmarks[16]. For each circuit, at least one erroneous output has been found by pre-simulation. Design error location is only applied for the selected erroneous output. Table 5 gives the erroneous output and some statistics for each circuit. All the terms are given with respect to the erroneous output.

For the output 04 of the circuit SIGNET, the difference function Dif $=1$. it means that all the combinations of the inputs are the distinguishing patterns. Therefore, the design error must be located at the output 04. Table 6 shows the experimental results for these circuits. The experimental results show that our design error locating method can locate the design error in one signal line for the experimental circuits.

Table 4: The design errors lists

\begin{tabular}{|c|c|}
\hline Circuits & The type of design error \\
\hline LC-CUNTT & A substitution of an OR gate for an AND gate at gate t32 \\
\hline SIGNET & An extra inverter at the output O4 \\
\hline C1355 & A missing inverter at the fan-out branch \{I20-t644\} \\
\hline C432 & A substitution of an AND gate for a NOR gate at gate t151 \\
\hline C880 & A missing fan-in t161 at gate t171 with fan-ins t161 and t165 \\
\hline C1908 & An extra inverter at the fan-in t108 of gate t65 \\
\hline
\end{tabular}

Table 5: Statistics of benchmark circuits

\begin{tabular}{|c|c|c|c|}
\hline Circuits & Erroneous output & No. of signal lines & No. of dis. patterns \\
\hline LC-CUNIT & O14 & 80 & 6 \\
\hline SIGNET & O4 & 198 & $*$ \\
\hline C1355 & O1 & 1154 & 1048576 \\
\hline C432 & O5 & 269 & 72579 \\
\hline C880 & O9 & 118 & 512 \\
\hline C1908 & O9 & 1035 & 248584 \\
\hline
\end{tabular}

Table 6: Experimental results for design error location

\begin{tabular}{|c|c|c|c|c|}
\hline & \multicolumn{2}{|c|}{ Fault simulation } & \multicolumn{2}{c|}{ Final results } \\
\hline Circuits & Simulated patterns & Candidates & CPU time(s) & Candidates \\
\hline LC-CUNIT & 6 & 20 & 0.40 & $\mathrm{t} 32$ \\
\hline SIGNET & $*$ & $*$ & 0.06 & $\mathrm{O} 4$ \\
\hline C1355 & 1087 & 14 & 35.82 & {$[120-\mathrm{t} 644]$} \\
\hline C432 & 168 & 5 & 10.0 & $\mathrm{t} 151$ \\
\hline C880 & 85 & 3 & 0.04 & $\mathrm{t} 165 / \mathrm{t} 171$ \\
\hline C1908 & 212 & 6 & 24.2 & $\mathrm{t} 108$ \\
\hline
\end{tabular}




\section{Conclusions}

For an incompletely specified function, the method to compute the corresponding 3 terminal BDD has been described. We also showed one application of 3-terminal BDDs for the verification of two incomplete functions. For locating the sources of design errors that exist in a gate-level circuit, a conditional stuck-at fault model was proposed, and a design error location method, based on fault simulation and released pattern generation, has been described. The experimental results showed that our method is efficient to locate the single design error in incorrect circuits.

\section{References}

1. M.S. Abadir, J. Ferguson, T.E. Kirkland: Logic Design Verification via Test Generation. IEEE Trans. on CAD Vol. 7, No. 1, Jan. 1988, pp. 138 - 148

2. G.D. Hachtel, R.M. Jacoby: Verification Algorithms for VLSI Synthesis. IEEE Trans. on CAD, Vol. 7, No. 5, MAY, 1988, pp. 616 - 640

3. R.E. Bryant: Graph-Based Algorithms for Boolean Function Manipulation. IEEE Trans. on Computers Vol C-35, No. 8, Aug. 1986, pp. 677 - 691

4. S. Malik, A.R. Wang, R.K. Brayton, A. Sangiovanni-Vincentelli: Logic Verification Using Binary Decision Diagram in a Logic Synthesis Environment. Proc. ICCAD-88, 1988, pp. 6 - 9

5. S. Minato, N. Ishiura: Shared Binary Decision Diagram with Attributed Edges for Efficient Boolean Function Manipulation. Proc. 27th DAC, 1990, pp. 52 - 57

6. G. Odawara, M. Tomita, O. Okuzawa, T. Ohta, Z. Zhuang: A Logic Verifier Based on Boolean Comparison. Proc. 23rd DAC, 1986, pp. 208 - 214

7. K.A. Tamura: Locating Functional Errors in Logic Circuits. Proc. 26th DAC, 1989 pp. $185-191$

8. V. Pitchumani, P. Mayor, N. Radia: A System for Fault Diagnosis and Simulation of VHDL Descriptions. Proc. 28th DAC, 1991, pp. 144 - 150

9. S. Y. Kuo: Locating Logic Design Errors via Test Generation and Don't-Care Propagation. Proc. 1st EURO-DAC, 1992,pp. 466 - 471

10. M. Fujita, T. Kakuda, Y. Matsunage: Redesign and Automatic Error Correction of Combinational Circuits. Proc. IFIP TC10/WG10.5 Workshop on Logic and Architecture Synthesis, 1990, pp. 253 - 262

11. K.S. Brace, R. L. Rudell, R.E. Bryant: Efficient Implementation of a BDD Package. Proc. 27th DAC, 1990, pp. 40 - 45

12. N. Calazans, Q. Zhang, R. Jacobi, B. Yernaux, A.M. Trullemans: Advanced Ordering and Manipulation Techniques for Binary Decision Diagrams. Proc. EDAC92, 1992, pp. 452 - 457

13. S. J. Hong: Fault simulation Strategy for Combinational Logic Networks. 8th symp. Fault-tolerant Computing(FTCS-8), 1978, pp. 96 - 99

14. M. Abramovici, et al: Critical Path Tracing: An Alternative to Fault Simulation. IEEE Design \& Test of Computers, Vol. 1, No. 1, Feb., 1984, pp. 83 - 93

15.P. Goel: An Implicit Enumeration Algorithm to Generate Tests for Combinational Logic Circuits. IEEE Trans. on Computer, Vol. C-30, Mar., 1981, pp. 215 - 222

16. F. Brglez, H. Fujiwara: A Neutral Netlist of 10 Combinational Circuits. Proc. ICCAS85 